\title{
Flexible multi-node simulation of cellular mobile communications: the Vienna 5G System Level Simulator
}

\author{
Martin Klaus Müller ${ }^{1,2 *}$ D , Fjolla Ademaj ${ }^{1,2}$, Thomas Dittrich², Agnes Fastenbauer², \\ Blanca Ramos Elbal ${ }^{1,2}$, Armand Nabavi², Lukas Nagel ${ }^{2}$, Stefan Schwarz ${ }^{1,2}$ and Markus Rupp ${ }^{2}$
}

\begin{abstract}
The investigation and prediction of new trends and technologies for mobile cellular networks is of utmost importance for researchers and network providers to quickly identify promising developments. With the verge of the fifth generation of mobile communications (5G), networks become more and more heterogeneous and dynamic while the amount of active users within a cell keeps ever increasing. Therefore, the search for more efficient network layouts and configurations attracts massive attention while on the other hand becomes more and more complex. In this contribution, we present the Vienna $5 \mathrm{G}$ system level simulator, which allows to perform numerical performance evaluation of large-scale multi-tier networks, with numerous types of network nodes. The simulator is based on MATLAB and is implemented in a modular fashion, to conveniently investigate arbitrary network and parameter constellations, which can be enhanced effortlessly. We first discuss the distinguishing aspects of our simulator platform, describe its structure, and then showcase its functionality by demonstrating the key aspects in more detail.
\end{abstract}

Keywords: Mobile communications, 5G, System level simulations, Cellular networks, Heterogeneous networks, Propagation models

\section{Introduction}

System level (SL) simulations have become indispensable for investigating and predicting the performance and behavior of wireless cellular systems [1-3]. The main interest of SL simulations thereby lies in network-related aspects, such as performance evaluation of key network parameters (e.g., capacity, latency, coverage, efficiency), rapid prototyping of novel methods and features (e.g., resource allocation, mobility management, multi-point coordination), and network planning [4-7]. SL simulations are unchallenged for understanding the potential of novel technologies in highly complex systems, such as cellular networks, with relatively modest implementation overhead and are therefore extensively utilized by both, academia and industry. The importance of SL modeling

\footnotetext{
*Correspondence: mmueller@nt.tuwien.ac.at

${ }^{1}$ Christian Doppler Laboratory for Dependable Wireless Connectivity for the Society in Motion, Vienna, Austria

${ }^{2}$ Institute of Telecommunications, TU Wien, Gusshausstraße 25/389, 1040 Vienna, Austria
}

and simulations is sometimes undervalued in scientific literature, since such methods can only provide numerical insights into the performance of the system. Yet, mathematically tractable analytic methods, such as those based on stochastic geometric tools $[8,9]$, often require application of restrictive assumptions and simplifications with respect to transceiver architectures, coordination capabilities of network nodes and modeling of the propagation environment. Furthermore, stochastic geometry is not able to represent network dynamics caused, for example, by the movement of users or the burstiness of data traffic and can therefore not address questions relating to mobility management and transmission latency. Despite analytic methods, the second main contender to SL simulations is field trials; yet, those are neither well suited to early stages of the development process of novel technologies, due to the cost and time effort required, nor can they easily be performed on a large scale without significantly impairing the performance of live operating mobile networks. 
SL simulations encompass a large number of network elements and thus require the consideration of many interconnecting (desired and interfering) transmission links. Correspondingly, handling computational complexity becomes a major concern. A widely accepted solution is the application of link abstraction models that specify the interaction between LL and SL simulators [1]. As the fifth generation of mobile communications (5G) introduces a multitude of novel concepts and contender technologies, such as full-dimensional (FD)/massive multiple-input multiple-output (MIMO) beamforming [10-12], mixed numerology multicarrier transmission $[13,14]$, non-orthogonal multiple access by superposition transmission $[15,16]$, and transmission in the millimeter wave (mmWave) band $[17,18]$, there is a need to revise and enhance existing modeling and simulation approaches. Our research group at the Institute of Telecommunications at TU Wien has been active in the field of LL and SL simulation of cellular networks already since the introduction of Long-Term Evolution (LTE) in late 2008. We developed in the past a whole suite of LTE compliant LL and SL simulators [19-21], known as the Vienna LTE simulators, which we successfully share with other researchers to facilitate reproducibility in wireless communications academic research [22, 23]. Evolving these simulators, however to support the increased heterogeneity and the emerging use cases of $5 \mathrm{G}$, is not straightforward due to a lack of flexibility of the simulation platform in terms of implementation structure and functionality. This was the reason for us to develop the two newest members of the Vienna Cellular Communications Simulator (VCCS) suite [22], namely the Vienna 5G LL simulator and the Vienna 5G SL simulator. The description of the latter is the scope of this publication.

The Vienna SL simulator (Vienna 5G SL simulator) explicitly models the network geometry and thereby allows, in combination with several propagation models for various propagation effects, to simulate the network performance based on signal strength and accumulated interference, combined in the signal to interference and noise ratio (SINR). Thus, the scope is on flexible generation of networks with arbitrary geometry and several types of base stations (BSs) and users, which also enables to simulate multi-tier networks. Since also complex and sophisticated channel models are available, advanced transmission modes, such as FD MIMO, can be investigated.

Several abstraction steps are necessary to actually perform system level simulations. The physical (PHY) layer transmission is abstracted via input from the Vienna SL simulator (Vienna 5G LL simulator) (cf. Section 3). While the medium access control (MAC) layer is represented by scheduling and feedback functions, we omit the implementation of the complete protocol stack. These abstractions and the generally efficient object-oriented programming (OOP)-based implementation allow to simulate large-scale networks with several thousands of active network nodes.

The Vienna 5G SL simulator is implemented in MAT$\mathrm{LAB}$ and is available for download under an academic use license [22]. This allows on the one hand reproducibility of research results and on the other hand it provides a unified tool for academic researchers to pursue their own research ideas. This is facilitated by the modular, OOPbased structure that allows to adapt parameters freely and exchange and enhance functionality also by, e.g., adding new propagation models.

\section{Scientific contribution}

In this chapter, we discuss the novelty of our simulator and state its unique abilities. Therefore, we first compare it to already existing simulation tools and then highlight the scientific contribution and novelty of our simulator. Most of these highlights will be showcased in more detail in Section 4.

\subsection{Related work-existing simulation tools}

Since SL simulators are convenient tools to investigate the network performance, naturally, there is a multitude of them available. The popularity of SL simulators can already be seen from the amount of existing simulators for Long-Term Evolution-Advanced (LTE-A). Next to the Vienna LTE-A SL simulator exists LTE-Sim [24] or SimuLTE [25] to name two examples with an academic background. Both simulators however only provide LTE-A functionality and do not offer extensions for $5 \mathrm{G}$. Generally, we distinguish between simulators with an academic background on the one side and from industry on the other side. The former usually includes more features than defined in the standard to support research and are mostly freely available to other researcher (under an academic-use license). Simulators from industry, on the other hand, are commonly only available for paying customers and mostly restricted to standardized features. For this reason, we compare our Vienna 5G SL simulator only to other freely available simulators and tools with an academic background.

Popular simulation tools are discrete-event network simulators, such as $\mathrm{OMNeT}++$ or ns-3. While these are basically generic network simulators, there exist dedicated modules for the simulation of LTE and 5G-based networks $[25,26]$. Due to the event-driven nature of these simulators, they are packet oriented and model large parts of the protocol stack. This, however, makes it prohibitive to simulate very large networks with many network nodes in terms of computational complexity. 
The OpenAirInterface [27] is an open source platform for the simulation of wireless networks. As of this writing, it implements the 3rd Generation Partnership Project (3GPP) LTE Rel. 8 and parts of later releases. It implements the full protocol stack and provides an interface to hardware platforms. Additionally, also the core network is integrated in this platform, which allows to investigate aspects of future radio access network (RAN) technologies. Even though the realism of simulation results is improved by integrating the core network, the increased complexity limits the number of network nodes in one simulation. Thus, it is rather to be categorized as a network simulator than a SL simulator.

There are several publications that claim to describe a 5G SL simulator. However, the corresponding simulators are either not available to others [28] or they are simple derivations of the Vienna LTE-A SL simulator that include some $5 \mathrm{G}$ aspects $[29,30]$. The simulator described in [31] is again event driven and only combines LTE-A and wireless local area network (WLAN) protocol stacks as a 5G feature.

One drawback of network simulators, which implement the entire protocol stack, is their large computational complexity, which makes it difficult if not impossible to utilize them for large-scale SL simulations. By focusing our efforts on highly adjustable network generation, including multi-tier networks and arbitrary network geometry, and by providing a large spectrum of propagation models with the possibility of effortless inclusion of further methods, the Vienna 5G SL simulator constitutes a powerful tool for SL simulations for $5 \mathrm{G}$ and beyond.

\subsection{Scientific contribution and novelty}

The need for a successor of the Vienna LTE-A SL simulator stems from the new requirements for the simulation of $5 \mathrm{G}$ networks $[3,32]$. During the implementation of our simulator, we made sure that the code structure grants flexibility and scalability for defining new simulation scenarios by combining and adding new functions (e.g., propagation models, scheduling algorithms) and expanding them (e.g., new user types, multi tier networks). Thanks to the modular code structure, the Vienna 5G SL simulator is prepared for emerging scenarios and applications, even beyond the scope of 5G. In the following, we detail the features that make our simulator fit for the simulation of $5 \mathrm{G}$ networks and we discuss the general advantages of our simulator.

\section{$5 G$ features:}

- Flexible implementation: The timing and frame structure can be adapted arbitrarily. This allows for defining resource grids for different subcarrier spacings, making it possible to adapt to requirements from different standards, as well as supporting flexible numerology use cases as defined in 5G [33].

- Propagation models: We support numerous propagation models for path loss, shadowing, or antenna patterns. Large efforts have been made for $5 G$ to define new, appropriate channel models, which we support directly, such as the 3GPP 3D channel model [34], the extended 3GPP 3D channel model for $\mathrm{mmW}$ ave and carrier frequencies up to $100 \mathrm{GHz}$ [35], and the recently published 3GPP 3D channel model for aerial devices [36]. Furthermore, by providing a channel interface it is possible to incorporate different outsourced channel models as for example the Quasi Deterministic Radio Channel Generator (QuaDRiGa) channel model [37].

- Heterogeneous networks and geometry: A huge emphasis in $5 \mathrm{G}$ is on the increased heterogeneity of networks. We therefore provide an arbitrary amount of individually defined BS and user types. These can be placed freely in the region of interest (ROI) and can also change their location over time, according to a predefined route or a random movement pattern. Additionally, the path loss model is adapted to the state of the corresponding link, according to line-of-sight (LOS)/non line-of-sight (NLOS) conditions or BS type. This can also be influenced by blockage objects, such as buildings or walls, which can freely be placed in the simulation area.

- D2D and IoT scenarios: Our simulator allows the evaluation of up-, down- and side-link in one simulation. Thus, device-to-device (D2D) as well as vehicle-to-everything (V2X) or relay scenarios can be investigated in our simulator. In the context of Internet of things (IoT), many (active or inactive) network nodes have to be considered in the same scenario at the same time. Thanks to our efficient implementation, our simulator can handle several thousand users in a multi-tier network.

\section{General simulation features:}

- LTE-A functionality: Regarding timing, frame-structure, and transmit modes, the Vienna 5G SL Simulator provides backwards compatibility to its LTE-A-based predecessor, the Vienna LTE-A SL simulator.

- Efficient implementation: In order to minimize the simulation time, we took several measures to reduce the simulation overhead and to increase the general efficiency of the code. We investigated means of optimizing the aggregate interference, such that results are not flawed at the border of the ROI. Additionally, we pregenerate all possible variables before the actual simulation loop, e.g., the whole 
network geometry and the channel traces. This is done in such a way that individual chunks of the simulation remain independent of each other, which allows for running these chunks in parallel on a multi-core system or even a simulation cluster.

- Precise geometry: A common approach for the calculation of macroscopic fading (MF) values in system level simulations is to use maps with finite resolution (see, e.g., Claussen maps [38]) and to assign always the closest available value to the actual user or BS position. This is, on the one hand, convenient for precalculating these values for a finite number of positions; on the other hand, it creates a large overhead and leads to imprecise results due to the finite spatial resolution. We completely avoid the calculations of maps and only utilize precise locations. Especially, the generation method for correlated shadow fading had to be enhanced for this approach, for which we use a new generation method that allows to calculate values for arbitrary positions [39].

- Input from Vienna 5G LL simulator: Due to the abstraction of the actual transmission by signal to noise ratio (SNR)-to-block error ratio (BLER) mapping curves for specific modulation and coding schemes (MCSs), our simulator benefits from the input provided by the Vienna 5G LL simulator. For different modulation orders or coding schemes with variable blocklength, these curves can be produced in the LL simulator and are then directly used for SL simulations.

\section{Simulator structure}

To better understand how the aforementioned features are represented in our simulator structure, we provide here an overview thereof. The scope of this document is to give an idea of the simulator's capabilities but not to explain its functionality in all details. For a more thorough explanation, please refer to the user manual and detailed feature list, which can be found on our simulator homepage [22].
As already mentioned, the main focus of the Vienna 5G SL simulator is on investigating large-scale networks based on their geometry and including defining propagation effects by appropriate models. The most important metric is the SINR, which accumulates the attenuated signal from the desired transmitter and all interferers at the receiver location. This metric depends on the noise level and more importantly on the user and BS constellation and the utilized propagation models for each link. Average performance results are obtained through Monte-Carlo simulations, by averaging over spatial and temporal realizations. The former are represented by variations in the network geometry and the modeling of MF values; the latter are included through the variations of channel coefficients over time. Based on the individual SINR results, scheduling decisions are made, and further metrics can be deducted, such as average user throughput or cell load.

The simulator structure is designed for flexibility and grants this through modular architecture, where individual functions can be exchanged independently. Coarsely, the simulator is partitioned into four parts: initialization, pregeneration, the main simulation loop, and postprocessing. This is also shown in Fig. 1 including the most important steps performed in each individual block.

\subsection{Initialization}

Before beginning a simulation, all necessary parameters have to be defined. We provide a number of predefined scenarios that showcase the capabilities of our simulator and demonstrate the existing feature choices. Based on these, first initializations in a next step so-called dependent parameters are set, most importantly the time line of the simulator. This is an integral concept of our simulator, to divide the timeline into time slots (TSs), segments, and chunks (cf. Fig. 2).

A TS is the time unit based on which scheduling is performed (and therefore is the time unit spent in one iteration of the inner simulation loop). It is a fixed value, e.g., $1 \mathrm{~ms}$ to represent LTE-A subframes, but can be freely

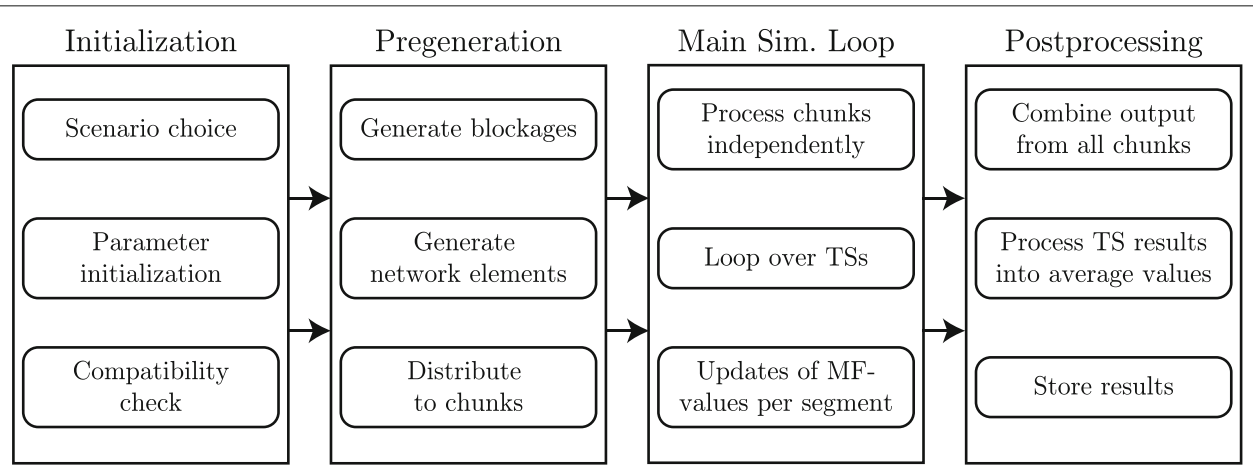

Fig. 1 Structure overview of the Vienna 5G SL simulator. The simulator is divided into four major blocks. In the figure, the main tasks that are performed per block are displayed 


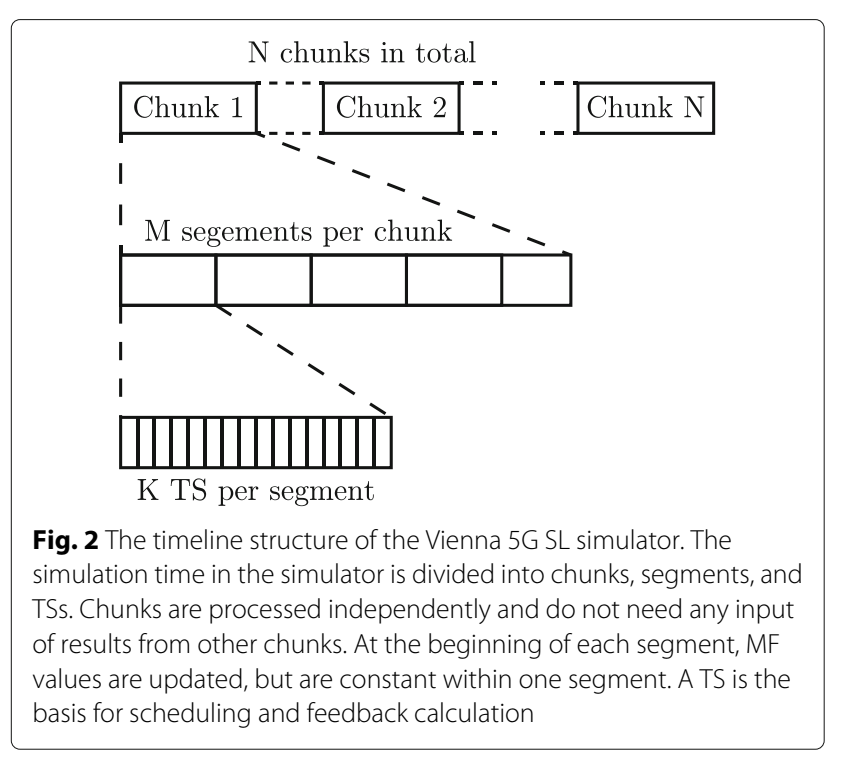

specified. A segment is defined as the time span for which the values of the MF are assumed to be (approximately) constant-they are only updated once per segment. Thus, also the BS assignment (based on received signal power or SINR) is unchanged for the length of a segment. In the initialization phase of the simulation, the length of a segment is determined based on the speed and trajectory of the users and the specified decorrelation distance. This implies that for stationary users, only one segment is created and MF values are only calculated once. A chunk is a sequence of consecutive segments. As indicated in Fig. 2, a considerable amount of time (that is not simulated, but used for trajectory generation) passes between chunks, such that the position of mobile users changes significantly between chunks. Irrespective of mobility, it is assumed that the scattering environment changes significantly between chunks, and thus, channel coefficients are uncorrelated. Since the input into each chunk is sufficient to perform all computations and no generated result from another chunk is needed in any chunk, it is possible to separate them and process them in parallel. Due to this preparation step, distributing individual chunks to parallel threads is possible without further effort, since all chunks can be processed independently from each other.

At the end of this stage, the specified parameters are checked for compatibility in order to avoid any issues or inconsistencies during the simulation.

\subsection{Pregeneration}

In the next part of the program, all elements with geometric location are created. All positions are specified in $\mathbb{R}^{3}$, but there is the option to discard the third dimension in order to perform two-dimensional simulations, thereby saving computational complexity. If specified by the selected scenario, in a first step, walls and buildings are placed within the network area (cf. Section 4.1.2).

Afterwards, BSs and users are generated according to the previously defined parameters. BS objects themselves do not have a geometric location, but contain one or more antenna elements. This allows to simulate distributed antenna systems (DASs) and remote radio heads (RRHs). While it is in general possible to allow movement for all network nodes, we assume for now that BS positions are constant over time. Users, on the other hand, can change their position in each segment according to their predefined trajectory. At this point, along with the network elements within the ROI, an interference region is created, which emulates interference from outside the actual ROI (cf. Section 4.2.1).

When the pregeneration step is finished, the necessary information for each chunk is combined. Only the corresponding values are bundled here, such as the user positions valid for the individual chunks. Thus, we reduce the overhead of data to hand over to the main simulation loop, but still provide all information that is required to perform the simulation within each chunk, thus separating them and enabling parallel simulation.

\subsection{Main simulation loop}

After distributing the configuration to the individual chunks, they are processed individually-consecutively or in parallel, dependent on the simulation configuration. Each chunk contains a loop over TSs. Before every iteration of this inner loop, it is checked if the current TS marks the beginning of a new segment. If that is the case, the positions of all users are updated, as well as the corresponding MF values with respect to the desired transmitter and all interferers. Respective values are distancedependent path loss, antenna gain, BS assignment, and link condition (cf. Section 4.1.2). For a simulation with uplink and downlink, these steps are performed once per TS (for frequency division duplex (FDD)) or only in alternating TSs for time division duplex (TDD). In Fig. 3, we present an overview of this main simulation loop.

The inner loop over TSs contains five major steps. First, the channel is updated for all links, according to the channel model. Which channel model types are supported and how they are implemented is described in more detail in Section 4.1.1.

Then, the scheduling is performed. Also for the scheduler several types are available, such as a classic round robin scheduler or a best channel quality indicator (CQI) scheduler, but also user-defined functions can comfortably be added here. According to the respective scheduling metric, users are assigned to transmit on the available elements in the resource grid. Once this is done for all BSs, the link quality model (LQM) is called. This is one of the major abstractions for our SL simulator, since 


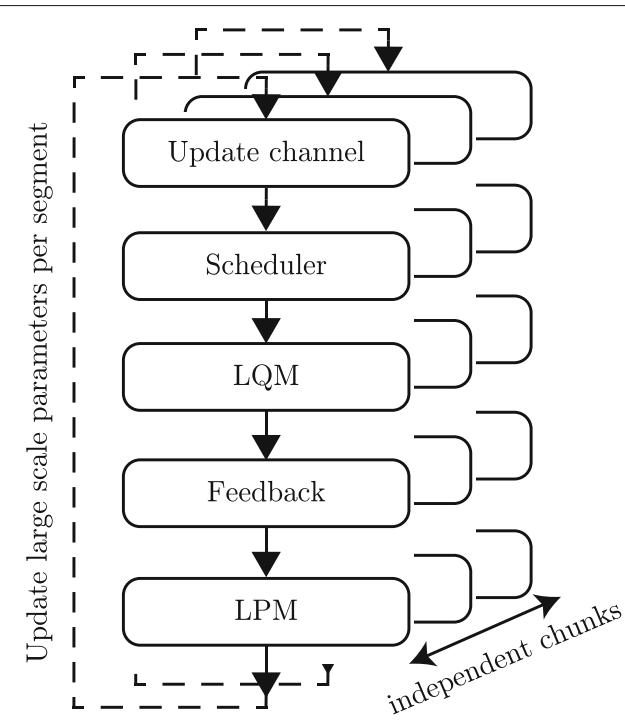

Fig. 3 Main simulation loop of the simulator. The main loop consists of an outer loop over chunks that are processed independently. At the beginning of the inner loop over TSs, the MF values are updated if a new segment begins. For each iteration, five steps are performed, which are updating the channel (i.e., the small-scale fading), distribution of the available resources to users by the scheduler, calculation of the postequalization SINR in the LQM, calculation of feedback, and finally mapping of SINR to BLER in the LPM

coding, modulation, channel, and equalizer are combined into a postequalization SINR value, which represents the combined performance of the physical layer transmission (cf. [40]).

In the next step of the main simulation loop, the feedback is calculated. There is a built-in, adaptable feedback delay, which is accounted for here. It is possible to utilize classical LTE-A-compliant implicit channel state information (CSI) feedback, consisting of CQI, rank indicator (RI) and precoding matrix indicator (PMI), or to utilize the unquantized channel coefficients as feedback, which is imperative for MIMO precoding schemes that require explicit CSI, such as zero forcing (ZF) multi user MIMO (MU-MIMO) transmission [41, 42]. Here, it is important that the scheduler can handle the generated feedback, i.e., the pair of scheduler and feedback function has to be aligned.

The second half of the abstracted transmitter-receiver chain, namely demapping and decoding, is combined together in the link performance model (LPM). The formerly calculated postequalization SINR is mapped to BLER and throughput, dependent on the MCS chosen for transmission. This is also the connecting point between the Vienna 5G LL simulator and SL simulator (cf. Section 4.2.3).

\subsection{Postprocessing}

When all chunks are processed, the individual results are combined. Here, a choice can be made on which values to save, in order not to bloat the finally stored file. This can again be adapted freely, such that only the results of interest are further processed.

From the individual results per TS and per BS and user, such as throughput or scheduling decisions, average values are calculated. These values range from average user throughput to average cell load or BLER, but also further metrics can easily be defined.

After this postprocessing step, the obtained results are displayed and stored in a file, together with the unprocessed, direct output for the individual TSs of the main simulation loop, if so desired.

\section{Detailed feature description}

In this section, we demonstrate the strengths of our simulator by discussing several implemented features in more detail. We split this section into two parts, namely features that are explicitly designed for simulation 5G networks and generally improved features that make the simulations more efficient and allow for more flexibility.

\subsection{Features for simulation of $5 \mathrm{G}$ networks \\ 4.1.1 Propagation models}

For SL simulations, propagation models are a key component, since they relate the geometry of the scenario to received signal strength and thus SINR, which is the main metric for further performance investigation. Within these models also lies the potential to acquire the necessary degree of abstraction; thus, including the relevant effects significantly impacts the value and accuracy of the obtained results [43]. There exists a multitude of models for various effects (see, e.g., [44]); additionally, appropriate models to account for novel developments within 5G standardization, such as transmission in the mmWave band and new BS types, are already being created.

Consequently, the Vienna 5G SL simulator provides a variety of options for macroscopic propagation effects (location dependent), such as path loss models, antenna patterns, or attenuation by blockages. Further models can be added conveniently, due to the modular structure of the simulator. The same is true for microscopic propagation effects, i.e., small-scale fading, which is generated according to a channel model. There has been considerable progress in defining more realistic models not only by adding temporal but also spatial correlation and by considering the third dimension for signal propagation. Due to the relatively complex procedure for these advanced channel models, we dedicate this section to explain in detail how different types of channel models are integrated in our simulator.

Realistic performance evaluation of advanced 5G techniques, such as FD-MIMO and three-dimensional (3D) beamforming, requires the use of a channel model that 
encompasses the main characteristics of large antenna arrays. In this regard, a channel model that makes use of geometric information, such as the angular spread in azimuth and elevation as well as physical location and orientation of antenna arrays at transmitter and receiver, is essential. This channel model type is known as geometrybased stochastic channel (GSC) model and is adopted by standardization bodies such as the 3GPP. The GSC model can be regarded as a balance between the two extremes of purely deterministic and purely stochastic channel modeling. While for purely deterministic modeling, i.e., ray tracing approaches, the simulated environment is completely deterministic, the GSC model reflects the statistics of a typical (urban, sub-urban, rural, etc.) environment. In contrast to purely stochastic modeling, which requires only the power delay profile (PDP) of the channel, the GSC model introduces a larger set of parameters dependent on both spatial- and time domain characteristics and determines the effective PDP based on positions of random scattering objects.

The Vienna 5G SL simulator provides a large variety of channel models that are classified in three basic types:

1. Stochastic two-dimensional (2D) channel models such as Rayleigh and Ricean fading, additive white Gaussian noise (AWGN), and classical PDP-based channel models such as Vehicular-A [45] or Typical Urban (TU) [46].

2. Geometry-based stochastic 3D channel models, such as 3GPP 3D channel model TR 36.873 [34], used for frequency ranges below $6 \mathrm{GHz}$ and 3GPP 3D mmWave channel model TR 38.901 [35] for frequency ranges up to $100 \mathrm{GHz}$.

3. Interface for outsourced channel models. We select QuaDRiGa channel model [37] as an example to show the simulator's flexibility and ease of integration.

The procedure to generate the actual channel coefficients is different for each of the three channel model types, as illustrated in Fig. 4.

For the first type, channel traces are generated in the PDP container, according to the appropriate channel model. We ensure that the temporal correlation of the generated channel traces matches the desired statistics of the channel model by applying the method proposed in [47]. The generation procedure of the second type of channel models and the channel interface are discussed below in more detail.

The 3GPP 3D channel model: This channel model consists of large-scale parameters (LSPS) and small-scale parameters (SSPS). The LSPs (e.g., shadow fading, Ricean $\mathrm{K}$-factor, delay spread, and angular spreads in azimuth and elevation) are position dependent and characterize each user location. These parameters are correlated based on

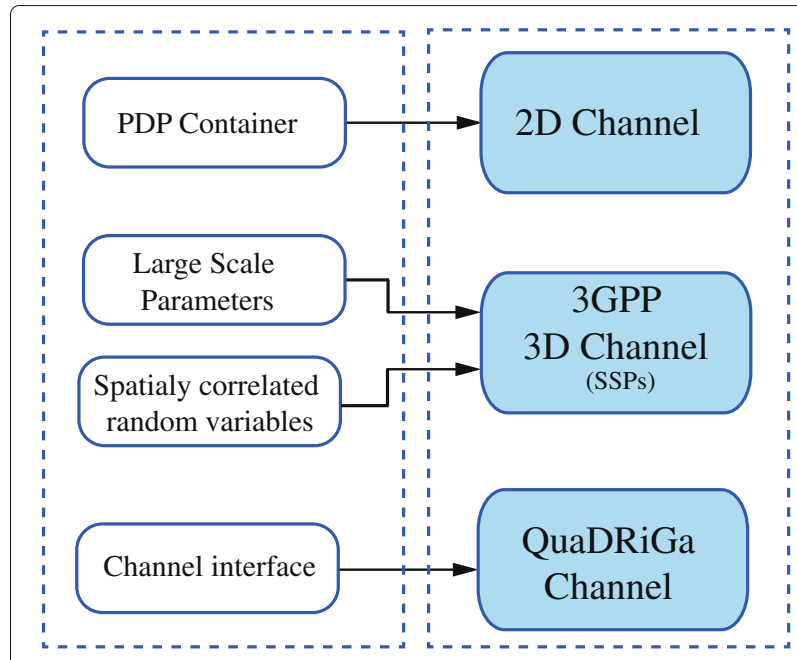

Fig. 4 Channel generation procedure. The channel generation process contains three different types of channel models: 2D channel models, 3D channel models, and a generic interface capable of incorporating outsourced channel models

exponential correlation functions in order to describe distance dependency of LSPs. The spatial correlation is sitespecific, meaning that it only accounts for links directed towards the same BS (uplink) or originating at the same BS (downlink). In the case of a sectorized BS, the correlation holds also for links of co-sited sectors. Correlated LSPs are generated in the main simulation loop after the user-to-BS assignment. The LSP correlation is performed independently for each chunk, while each of the parameters are updated on a segment basis. In this way, a segment is considered as a quasi-stationary region. This is in agreement with the parametrization of the decorrelation distance (see [48], Sec. 3.3.1), the only parameter used in the exponential correlation function. An example of spatially correlated LSPs is shown in Fig. 5. For each LSP, a map is generated only for the geometrical area of the links with a common BS, then correlated LSPs, denoted by $\sigma_{i}$, are extracted and saved for each link.

The generation of SSPs, as provided by 3GPP, consists of a step-wise procedure which includes several probability distributions that determine cluster- and rayspecific parameters as shown in Table 1. An important issue that arises at this step is the lack of spatial consistency since cluster- and ray-specific parameters $\left(D_{n}, S_{n}, X_{n}, Y_{n}, Z_{n, m}, \Phi_{n, m}\right)$ are generated for every user independently. We therefore extend the 3GPP compliant step-wise SSP generation (see [49] for a more detailed description) by including a new step to generate spatially correlated random variables based on the method proposed in [50]. This step is denoted as spatially correlated random variables and is applied before starting the actual generation of SSPs, as illustrated in Fig. 4. 


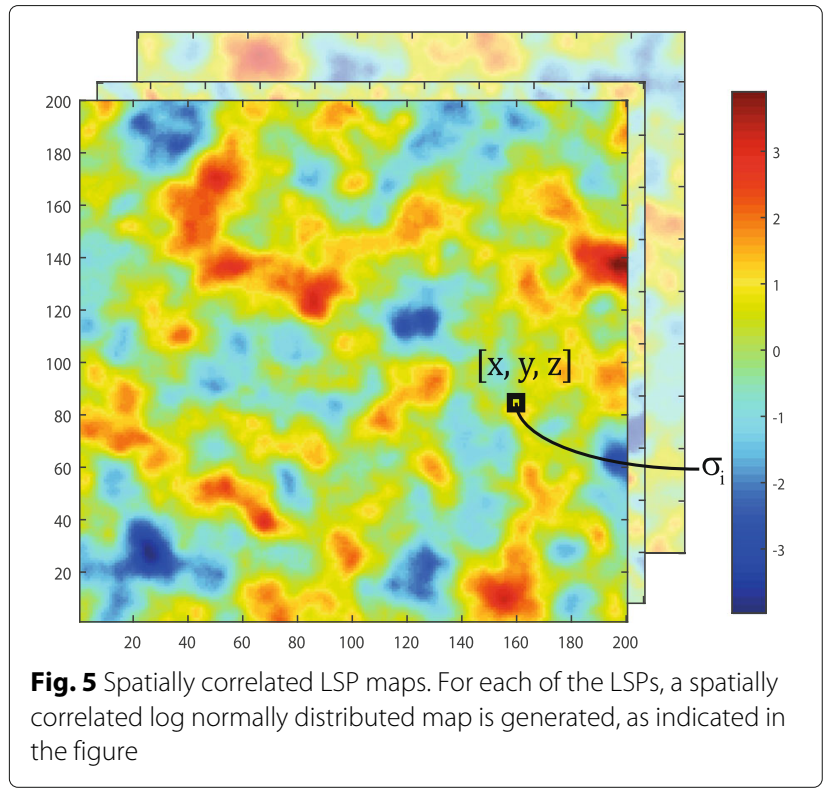

The spatially correlated random variables procedure starts by first generating one grid of independent and identically distributed (iid) random variables for each of the cluster- and ray-specific parameters. This grid is created for each chunk independently, and its size corresponds to the entire simulation area where the users are located. The resolution of the grid is determined by a defined parameter, $\Delta d$, representing decorrelation distance in meters. Afterwards, using bilinear interpolation, the corresponding values for each parameter and for every user location are calculated. This method will ensure that closely located users will experience similar angles of arrival/departure and other channel characteristics. Figure 6 shows an example of the parameter interpolation for two closely located users that lie within one grid. An advantage of this method is the ability to obtain the interpolated value for a specific user location with high accuracy. Another aspect of this procedure is that no maps are stored, grids are only generated for the purpose of obtaining the necessary values at the user positions and

Table 1 Probability distributions of random variables used to generate small-scale parameters as specified by 3GPP [34, 35]

\begin{tabular}{ll}
\hline Cluster $(n)$ and ray $(m)$ & Distribution \\
\hline Step 5: Delay & $D_{n}=\mathcal{U}(0,1)$ \\
Step 6: Shadowing term & $S_{n}=\mathcal{N}\left(0, \zeta^{2}\right)$ \\
Step 7: Angle sign & $X_{n}=\mathcal{U}(\{-1,1\})$ \\
Step 7: Angular variation & $Y_{n}=\mathcal{N}\left(0,\left(\sigma_{A S} / 7\right)^{2}\right)$ \\
Step 9: XPR & $Z_{n, m}=\mathcal{N}\left(\mu\right.$ XPR, $\left.\sigma_{X P R}^{2}\right)$ \\
Step 10: Initial phase & $\Phi_{n, m}=\mathcal{U}(-\pi, \pi)$ \\
\hline
\end{tabular}

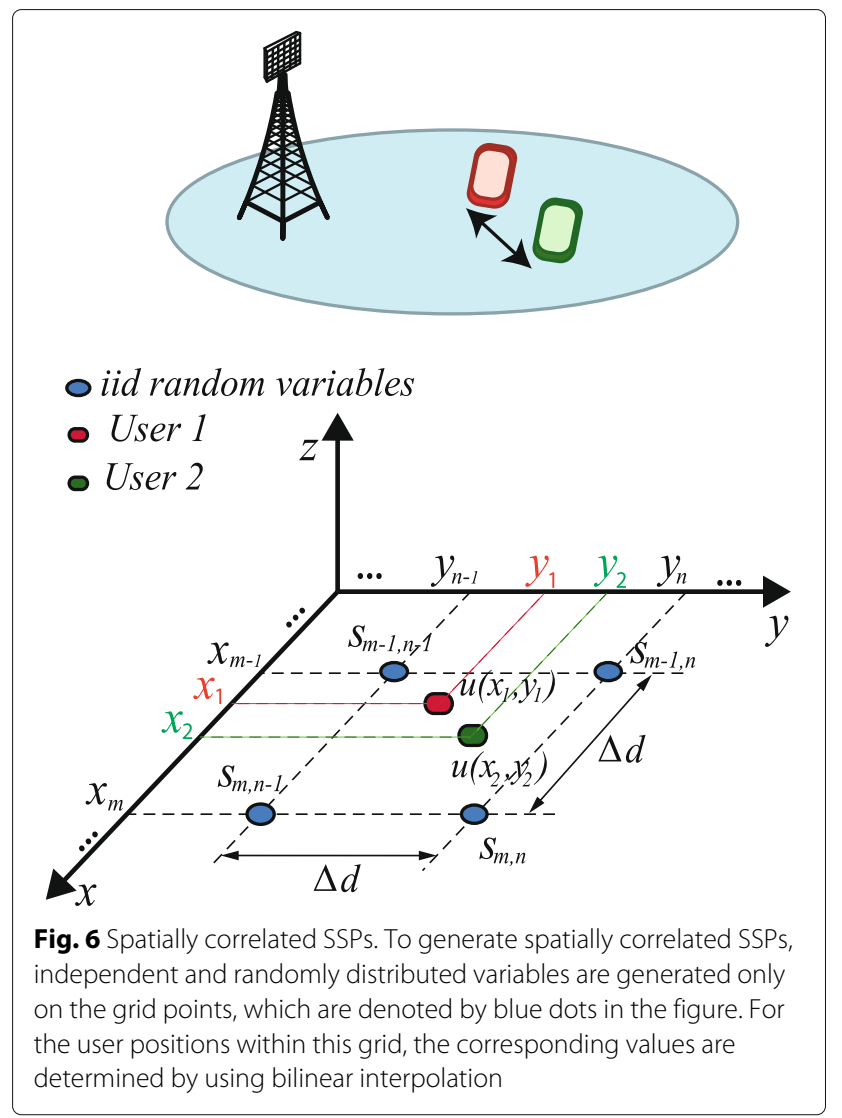

are deleted afterwards. Only the interpolated values are saved for every time slot. This is very important, as for large scenarios saving such grids can have huge impact on the memory efficiency of the simulator, a limitation that exists in our Vienna LTE-A SL simulator. More details about such precise geometry methods are discussed in Section 4.2.2.

Channel interface: The channel interface allows the usage of third party implementations of channel models. Currently, this interface is used to support the QuaDRiGa channel model. The QuaDRiGa source code itself is available at the website of the Fraunhofer Heinrich Hertz Institute (HHI). The QuaDRiGa channel model is calibrated according to TR 36.873 [34] and TR 36.901 [35] and supports carrier frequencies of up to $100 \mathrm{GHz}$. Additionally, the QuaDRiGa channel model is backed up by extensive measurement campaigns by the Fraunhofer HHI according to [35].

Simulation setups can be specified by using either the Vienna 5G SL simulator's syntax or QuaDRiGa's syntax. This makes the interface easy to use while at the same time allowing the user direct access to all of QuaDRiGa's features, such as antenna macros, that provide a set of predefined antenna arrays, ranging from simple omni or 
dipole antennas to more complex types as defined in [34] for the 3D model or in [35] for mmWave. The interface creates QuaDRiGa layout objects which are used to compute the channel coefficients. These coefficients are then properly rearranged and returned to the Vienna 5G SL simulator.

Further models can be supported in a similar fashion with some initial implementation effort. Therefore, the input from the simulator and the output of the model have to be processed appropriately for the specific model.

\subsubsection{Heterogeneous networks}

The flexibility and versatility of the Vienna 5G SL simulator especially shows in the broad variety of networks that can be created. BS and user types can freely be specified and placed within the scenario, which allows to simulate multi-tier networks that become more and more reality for actual deployments. This heterogeneity, which is a cornerstone of future $5 \mathrm{G}$ networks, is also reflected in the adaptive choice of path loss models, dependent on the link conditions.

BS and user types: The simulator supports various BS and user types in one simulation simultaneously. In the initialization phase of a simulation, properties that determine each BS and user type are specified. The BS types can be distinguished by different transmit powers such as macro, pico, and femto BSs. Furthermore, BSs can have different antenna types with specific antenna element patterns and different antenna array sizes.

Similarly, the simulator allows for different user types, e.g., pedestrians, cars, and machine-type users. User types are distinguished by several parameters, e.g., the movement pattern, the speed or the number of antennas. Figure 7 illustrates the BS association based on the maximum received power for a heterogeneous network consisting of macro, pico, and femto BSs as well as pedestrian and car users.

BS and user placement: Each BS is connected with one or more antennas for transmission and reception of signals, where each antenna has its own location in 3D space and represents a concentrated antenna array with potentially more than one antenna element. This enables simulation of DASs and RRHs and cloud RANs. Several predefined placement methods are available, ranging from random placement, e.g., according to a Poisson point process (PPP), or placement in a hexagonal grid with sectorized BSs; other methods can easily be integrated.

Equivalently for users, as the other endpoint of the connection, various spatial distributions can be applied, such as random placement following different random probability distributions, or predefined set of positions. Additionally, user positions can evolve over time. This happens either on predefined trajectories or random trajectories and depends on the velocity of the user.

The flexible definition of arbitrary BS and user types also prepares the simulator for important future concepts such as V2X, railway, or unmanned aerial vehicle (UAV). A V2X scenario is already included in Fig. 7 with a highway supplied by pico cells and a specific user type for car users. A railway can be defined in a similar manner, but with users confined in a defined space (potentially with an additional penetration loss into the train carriage) and with a predefined trajectory that corresponds to the train tracks. In order to model UAV scenarios, mostly the antenna pattern of the BS has to be adjusted. Therefore, also $3 \mathrm{D}$ antenna patterns are supported by the simulator.

Blockage modeling: The basic building block to represent signal blocking objects, is a rectangular wall with arbitrary dimensions and orientation in 3D. Buildings are created by combining five walls (four walls on the sides

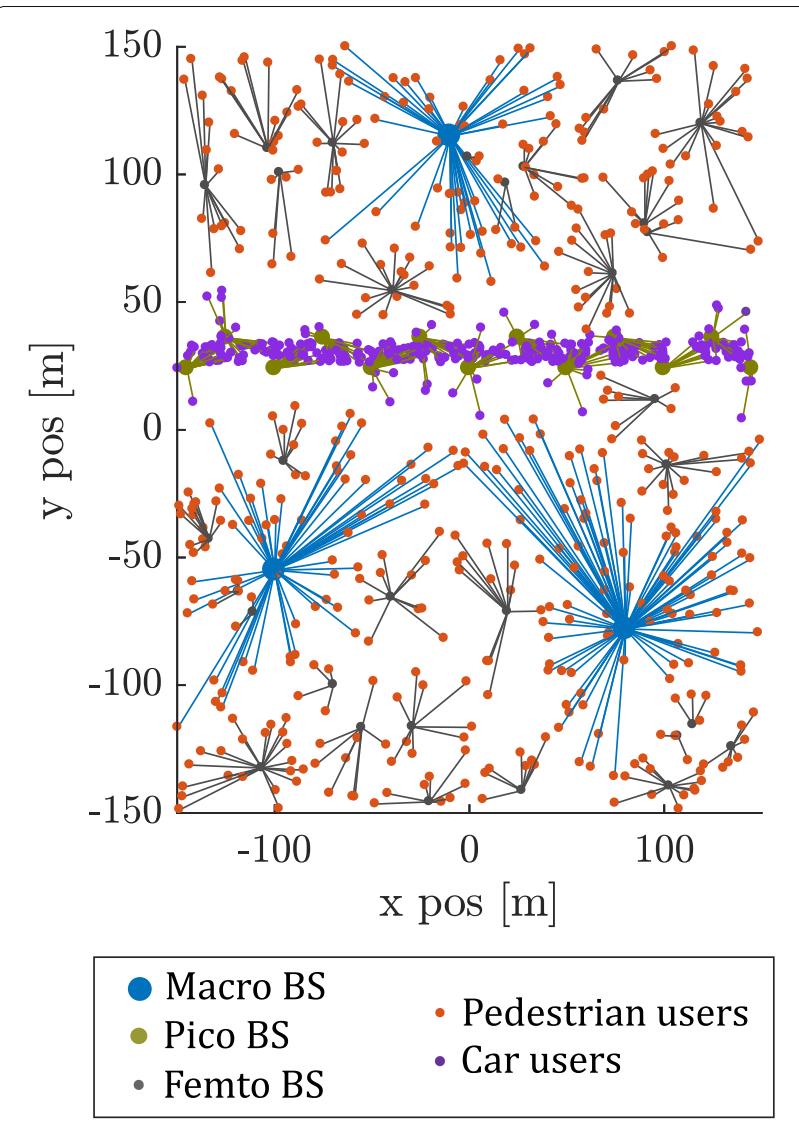

Fig. 7 BS-user association based on the maximum received power. This figure shows an example of a heterogeneous network consisting of various BS and user types. Colored lines denote the BS-user association, each color denoting a BS type. The implemented scenario represents an urban environment with one main highway going through the center, with dedicated pico cells for this region 
and a ceiling). With these basic elements, arbitrary city layouts can be generated, such as a Manhattan grid with streets and building blocks. Another option is incorporating real-world maps by extracting street and building data and importing them into the simulator to create appropriate blockage objects, similarly to $[51,52]$. These blockages are then used to determine the LOS or NLOS state of every link; additionally, blockages are used to switch between indoor and outdoor propagation models. Also investigations of the actual influence of blockages in a scenario can be performed, similar to [53]. To do so, blockages are assigned a penetration loss value, which can be the same for all walls, stem from a distribution of loss values, or can be predefined, which then allows to calculate the accumulated penetration loss along the link.

Situation aware path loss models: Since the simulated network can be highly heterogeneous, i.e., using different BS and user types and propagation environments, several situation-specific path loss models are in general utilized within one single simulation. The path loss models are specifically determined per link condition, i.e., BS type, indoor/outdoor user location, and LOS state, which requires to perform a large number of checks for every link in order to choose the appropriate path loss model. In order to avoid such overhead, we predefine a look-up table providing combinations of propagation conditions and scenarios, each related to a specific path loss model. A possible setup for link state and path loss models is shown in Table 2. In this example, the simulated network consists of macro BSs operating in low-frequency bands and femto BSs operating in the mmWave frequency band.

The look-up table can be extended without increasing the simulation complexity. This approach ensures a very flexible and situation aware selection of path loss models, which is well suited for the requirements of heterogeneous networks in 5G and beyond, since the heterogeneity not only stems from the actual network geometry, but also from the distinct behavior of the propagation for different situations within the scenario.

In order to identify the link status, the individual link condition has to be determined. While this is given for the BS type, there has to be a check for the LOS condition and for indoor or outdoor location of a user. For both conditions, there are three options available to determine the status. It can directly be inferred from the geometry of the scenario, when blockages are explicitly modeled. For LOS condition, it is simply checked, if any wall obstructs the link (cf. Fig. 8), while building information is used to identify if a user is located indoors or outdoors. Additionally, the two conditions can be modeled by considering a simple probability check for either case-representing the percentage of simulated area covered by buildings for the indoor decision or considering a distance-dependent LOS probability such as the one specified in the 3GPP 3D channel model for predefined scenarios. In order to account for spatial consistency, e.g., for avoiding that the condition for a group of user positions closely located, randomly jump from LOS to NLOS state, the indoor and LOS probability generation approach described in ([50], Sec. 2) and ([50], Sec. 3) can be used.

\subsubsection{Device to device communication}

D2D communications started to gather strength in the fourth generation of mobile communications with the development of safety critical applications and connected in-vehicle infotainment systems, and it is envisioned to keep growing in the fifth generation towards the direction of autonomous driving [54].

In this section we utilize D2D communication to enable relaying between users in order to improve the coverage in a microcellular urban network. We compute the performance of the direct link and the relay-assisted link in terms of SINR values, which are obtained with the lite simulation mode of the Vienna 5G SL simulator explained in Section 4.2.1. Our simulator is able to carry out uplink, downlink and sidelink transmission in one single simulation. Since most traffic and control signaling takes place in the downlink band, we consider the sidelink to be integrated into the uplink.

We create a 2D Manhattan grid scenario as shown in Fig. 9, where streets are distributed according to a Poisson line process with density $\lambda_{s}$. BSs and users are placed according to a PPP in every street with density $\lambda_{b}$ and $\lambda_{u}$ respectively. The path loss is modeled as a distance-dependent function similar to [55], where the path between transmitter and receiver is split into different segments depending whether the receiver is in the same street as the transmitter, in a crossing or in a parallel street. Users are attached to the BS BS that gives the minimum pathloss and are equally divided into two groups: relays and transmitters. Relays are idle users that have no data to send and therefore are available to convey the signal from the BS to other users. For that reason,

Table 2 An example of the look-up table used to determine situation aware path loss

\begin{tabular}{llll}
\hline BS type & In-/Outdoor & LOS/NLOS & Path loss type \\
\hline Macro & $\mathrm{O}$ & LOS & 3D-UMa LOS-O \\
Macro & $\mathrm{O}$ & NLOS & 3D-UMa NLOS-O \\
Macro & $\mathrm{I}$ & LOS & 3D-UMa LOS-I \\
Macro & $\mathrm{I}$ & NLOS & 3D-UMa NLOS-I \\
Femto & $\mathrm{O}$ & LOS & mmWave LOS-O \\
Femto & $\mathrm{O}$ & NLOS & mmWave NLOS-O \\
Femto & $\mathrm{L}$ & LOS & mmWave LOS-I \\
Femto & $\mathrm{I}$ & NLOS & mmWave NLOS-I \\
\hline
\end{tabular}




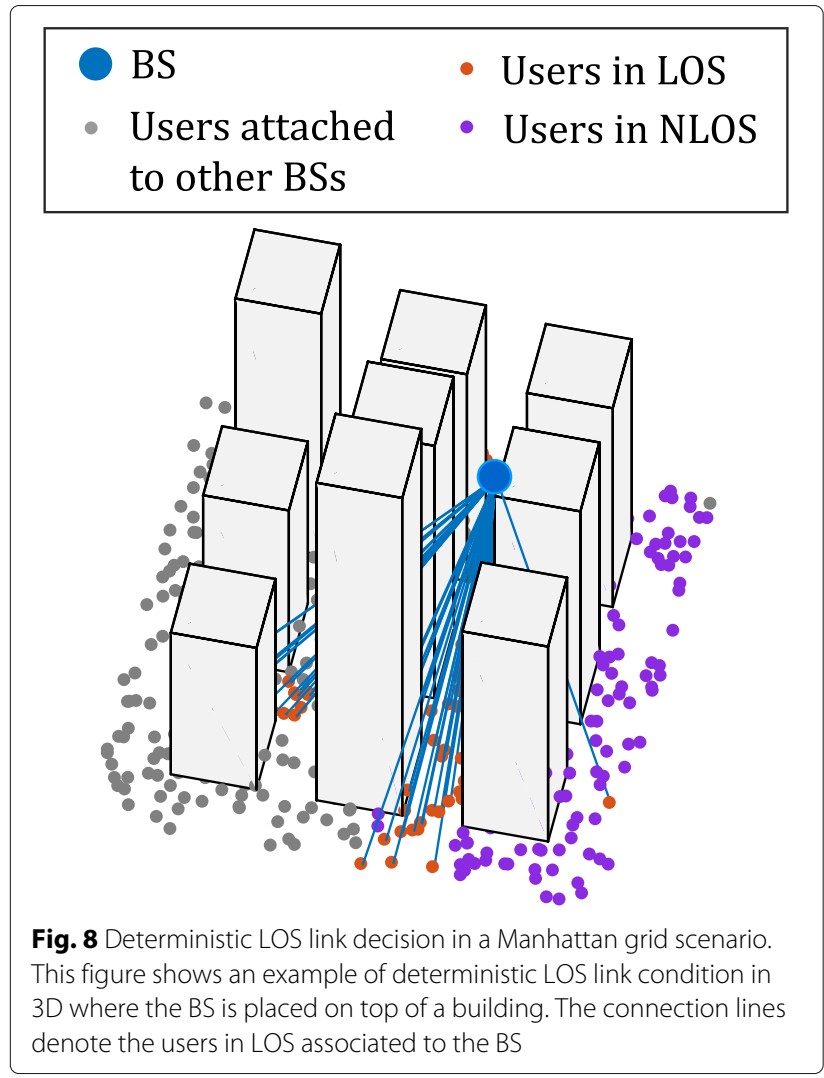

we consider that the selected relay should be within the same cell as the user. The communication between relays and BSs takes place in the downlink and uplink bands of the network. Besides, the communication among relays and users occurs in half duplex in the sidelink. In contrast to relays, transmitters have data to convey to their BS. Since the sidelink is integrated into the uplink, transmitters will interfere in the relay-user link. We consider that our attached BS performs orthogonal scheduling in the uplink band, that is, that interference from transmitters within our cell is avoided and therefore we consider only inter-cell interference in the relay-assisted link. The quality of the assisted link is defined by the link with the worst SINR of the BS-relay and relay-user link. In order to compute the SINR, we consider a noise spectral density of $-174 \mathrm{dBm} / \mathrm{Hz}$, a bandwidth of $10 \mathrm{MHz}$, and transmit powers $P T x_{b}=10 \mathrm{~W}$ and $P T x_{u}=0.2 \mathrm{~W}$ per BS and user, respectively. We assume a Rayleigh fading for each link.

With the scenario described above, we perform MonteCarlo system level simulations with 3000 samples each, for four different parameter combinations out of the set $\left\{\lambda_{s}, \lambda_{b}, \lambda_{u}\right\}$. The coverage probability of the direct and relay-assisted link over the user density is shown in Fig. 10. The coverage probability of the direct link is independent of the number of users and decreases as the BS density

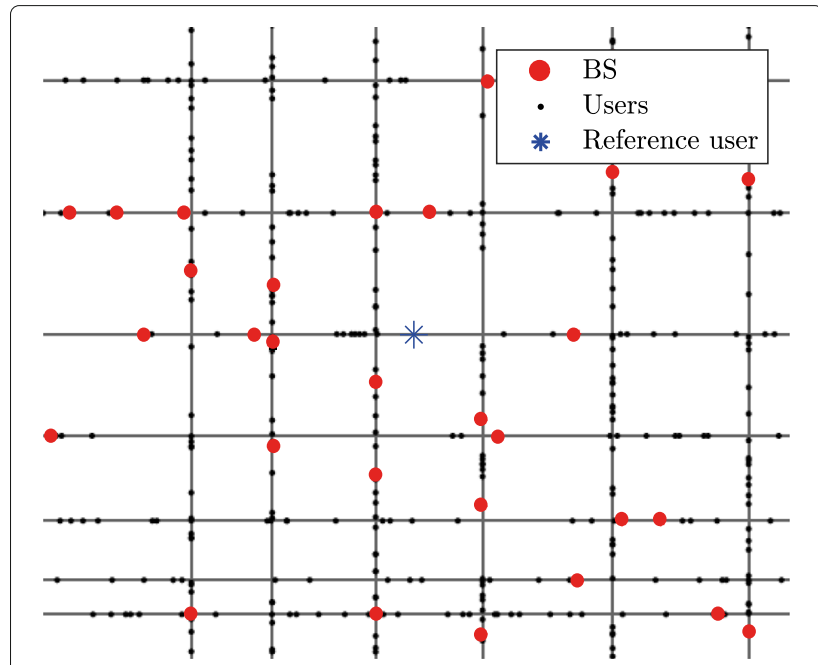

Fig. 9 Manhattan Poisson line process scenario. Example for a realization of streets, $\mathrm{BSs}$, and users

$\lambda_{b}$ increases since the interference from other BSs grows rapidly. Regarding the assisted link, for low user density $\lambda_{u}$, the improvement is almost negligible since the number of relays in the cell is very low or even zero. For high $\lambda_{u}$, the relay-assisted communication can improve the coverage probability of direct communication by almost $30 \%$.

\subsubsection{Internet of things}

With the introduction of the IoT paradigm and the trend of making everyday household objects able to communicate autonomously, future $5 \mathrm{G}$ networks will have to deal with a tremendously increased number of network nodes [56]. Even though the generated data packets tend to be small and required data rates are low, for some eventtriggered messages, the network will have to deal with a temporal traffic-spike.

From a simulation point of few, that means that a considerably larger amount of network nodes has to be handled simultaneously in one simulation, also due to the large concentration of IoT devices. Long generation cycles for packets require long duration of simulations and with relatively uninteresting results when the IoT devices are idle.

The timeline of the Vienna 5G SL simulator is separated into chunks that can be used to create a scenario that only simulates the time instances in which transmissions take place, without the overhead of simulating the time in between. Additionally, activity patterns can be defined to mimic the behavior of IoT devices. In this way, e.g., situations where event-driven messages flood the channel. Considering the simulation of large amounts of network nodes, we ensured that the Vienna 5G SL simulator is able 


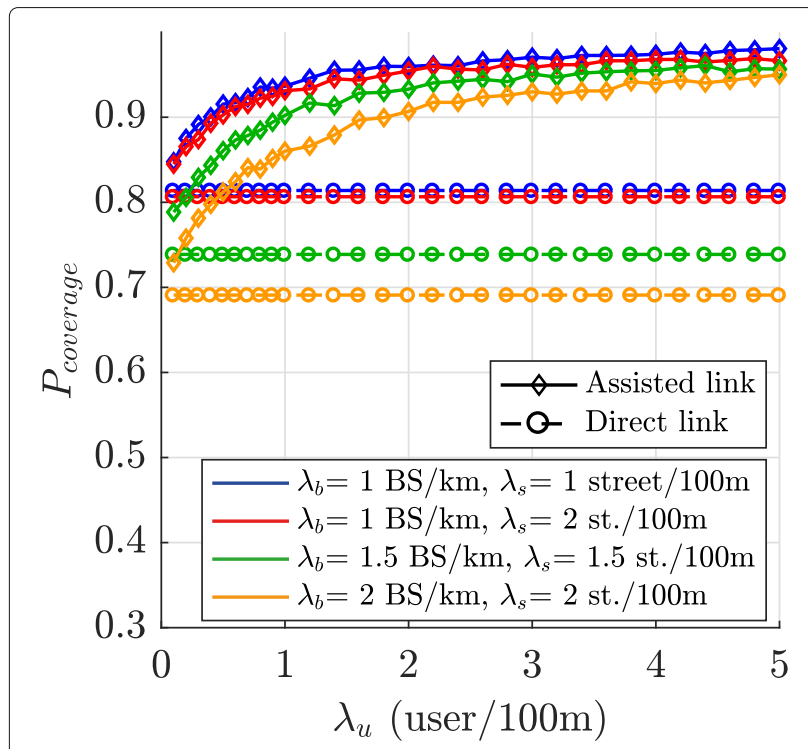

Fig. 10 Coverage probability. Direct link and relay-assisted link for different parameter sets over the user density with a SINR threshold of $T=0 \mathrm{~dB}$

to cope with this through efficient implementation and strict usage of OOP programming.

To illustrate this, we present an IoT scenario in Fig. 11 where more than 7000 users are simulated with the Vienna 5G SL simulator. Despite the huge number of network nodes, the simulation requires less than 16 GB RAM (which is usually the limiting factor here), for a total of 100 simulated TSs.

\subsection{Common system level features}

\subsubsection{General implementation enhancements}

To obtain meaningful average values from Monte-Carlo simulations, many realizations for scenarios with a large number of network nodes have to be simulated. Therefore, an efficient implementation is required, where redundant calculations and the generation of results that are either invalid or not of interest are avoided. This is ensured in the Vienna 5G SL simulator by a number of functionalities:

Lite simulation: When the simulator is used, e.g., for gaining insights into the coverage performance of a specific scenario, only the computed SINR values are of interest. Functions such as scheduling and the LPM are then not necessary. Therefore, the Vienna 5G SL simulator provides a lite version, where the SINR values are calculated from the network geometry and the chosen models, with simplified or no scheduler and LPM functionalities.

Parameter update: To assure that all links are simulated correctly, geometry dependent parameters need to be adapted when network elements move in the simulation area. At the same time, the recalculation of constant parameters has to be avoided for an efficient implementation. Thus the MF values (path loss, wall loss, antenna gain, shadow fading) and the link properties depending on them, e.g. LOS/NLOS, indoor/outdoor, are constant over a segment, while the small scale parameters (small scale fading) change for every TS and only keep their time correlation over a segment.

Interference region: At the border of the ROI, the lack of interference from outside of the ROI leads to an overestimation of SINR values. This effect can be mitigated by adding an outer simulation region that produces additional interference for the network elements close to the border. In the Vienna 5G SL simulator, the interfering nodes are simulated with a simplified scheduler and random feedback to limit the added computational complexity. Nodes are simulated as interfering nodes, if they serve no user, that generates simulation results.

Figure 12 illustrates an exemplary spatial base station distribution used for these simulations; it also illustrates that the relative overhead of simulating the additional interference region decreases with increasing size of the ROI. Thus, the efficiency of the simulation is enhanced, because more of the simulated area can effectively be used to generate results, as compared to the smaller ROI. Figure 13 shows how the additional interference region shifts the SINR distribution to lower, more realistic values, for a medium sized and a big ROI. For the efficient simulation of small ROIs, the Vienna 5G SL simulator will provide the option of a wrap around [57] to mitigate border effects in future releases.

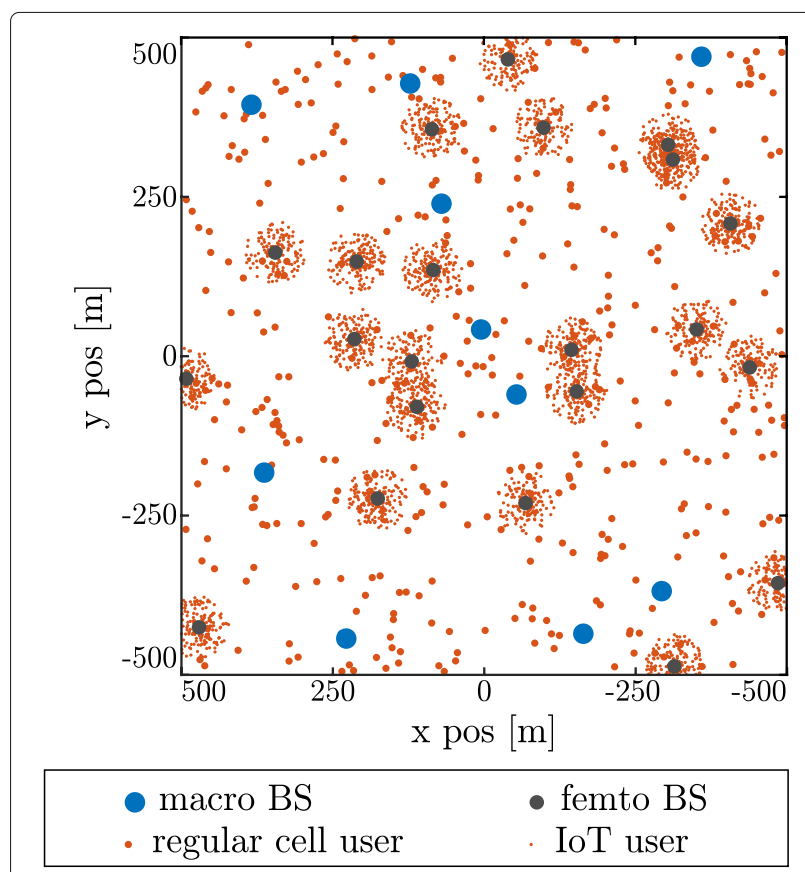

Fig. 11 Simulation of a large loT scenario. Network geometry with PPP distributed macro $\left(d=10 \mathrm{~km}^{-2}\right)$ and femto $\left(d=25 \mathrm{~km}^{-2}\right)$ BSs, regular cell users $\left(d=400 \mathrm{~km}^{-2}\right)$ and loT users $\left(d=25000 \mathrm{~km}^{-2}\right)$ distributed in circular clusters of radius $r=50 \mathrm{~m}$ around the femto BS 


\subsubsection{Precise geometry}

For SL simulator, there has to be a method that maps from the network geometry to LSP. As it was mentioned in Section 2, for shadow fading (SF) and path loss (PL), this is commonly implemented in form of maps, i.e., the ROI gets split into a set of pixels of a fixed size and thus, the SF and PL is represented by two arrays per BS. This method is chosen because the arrays with finite granularity are independent of the actual user positions and can thus be pregenerated. With the finite granularity of the array there arises the problem that the user positions have to be quantized to pixels, which could lead to a non-smooth behavior in the resulting curves. For example, consider a simulation with exactly one BS and one user that moves on a straight line from one BS to another. This user will experience jumps in its PL and some region in between the two BSs where the pathloss is equal. This behavior can artificially enlarge the assignment region for one BS while shrinking it for the other. The PL of this user is shown in Fig. 14. Obviously this problem can be tackled by increasing the resolution but then also the size of the arrays increase, which can have a severe impact on the memory efficiency of the simulator.

The Vienna 5G SL simulator pregenerates the movement trajectories of the users in the preparation of the simulation (cf. Section 3.1). Those position values are then used in the setup of every chunk to calculate PL and SF values for every position in floating point precision. For the PL, this comes naturally with the definition of most models, as they are only distance dependent but for the SF, this is not the case. In the Vienna LTE-A SL simulator, the method from [38] is used, which only allows the generation of correlated Gaussian values for arrays. In [39], we present an efficient way of generating $N$ dimensional arrays of correlated Gaussian values that is based on linear filtering. In the simulator, we restrict it to the two-dimensional case. The main steps of the method can be described as follows:

1. Let $r$ be an array that holds the first period of the periodic repetition of the desired autocorrelation,

2. Generate an array a of iid Gaussian variables

3. Calculate the spectrum $A:=\mathcal{F}\{a\}$,

4. Design the filter $H:=\sqrt{\mathcal{F}\{r\}}$,

5. Apply $H$ by element-wise (Hadamard) multiplication $B:=H \odot A$,

6. Calculate the spatial domain of $B, b:=\mathcal{F}^{-1}\{B\}$.

In this description, $r, a, A, H$ and $B$ are still maps with quantized positions that can be deleted as soon as the calculation of $b$ is finished. From the sixth step, it can be seen that $b$ need not be a map. It is possible to calculate instances of random variables for points that do not lie on the grid of quantized map-positions because the

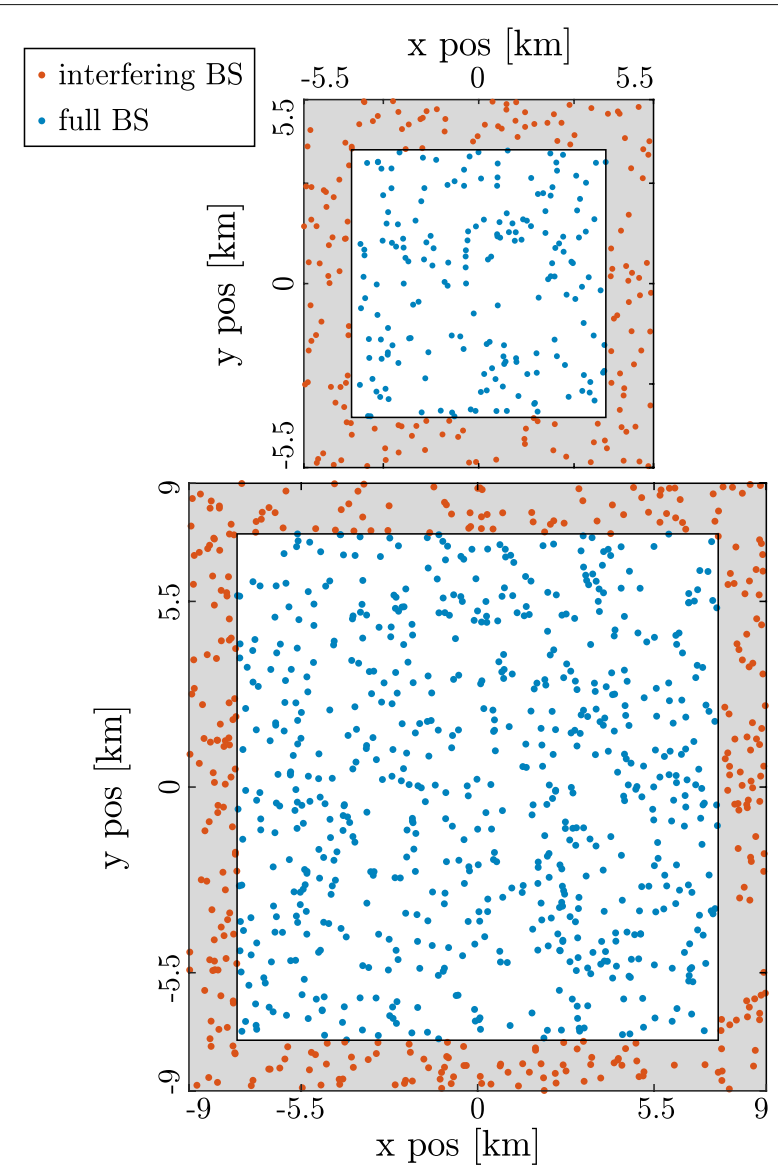

Fig. 12 Downlink simulation with interference region. This figure shows the base station in a small (upper) and big (lower) simulation region with an additional interference region (in gray). The absolute distance between $\mathrm{ROI}$ and the outer border of the interference region is constant for both scenario sizes

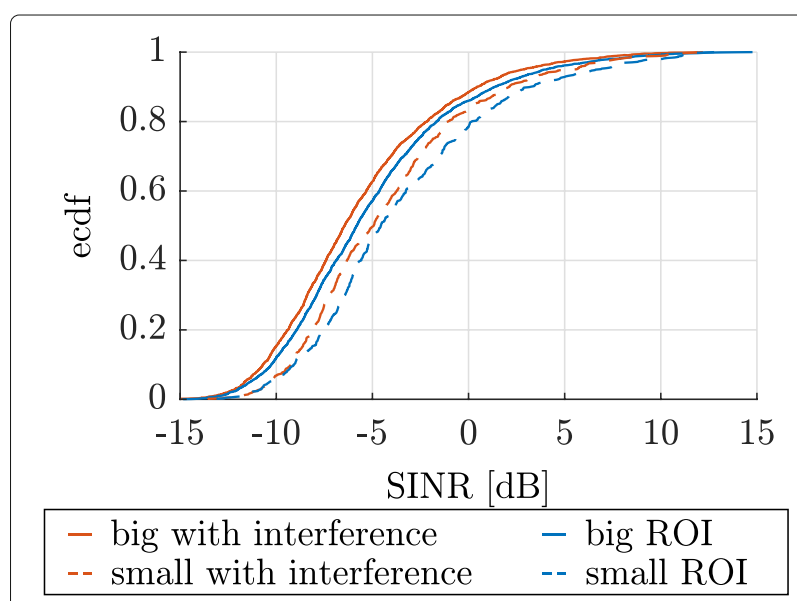

Fig. 13 SINR distribution with and without additional interference region. The SINR is overestimated in the scenarios without an additional interference region 


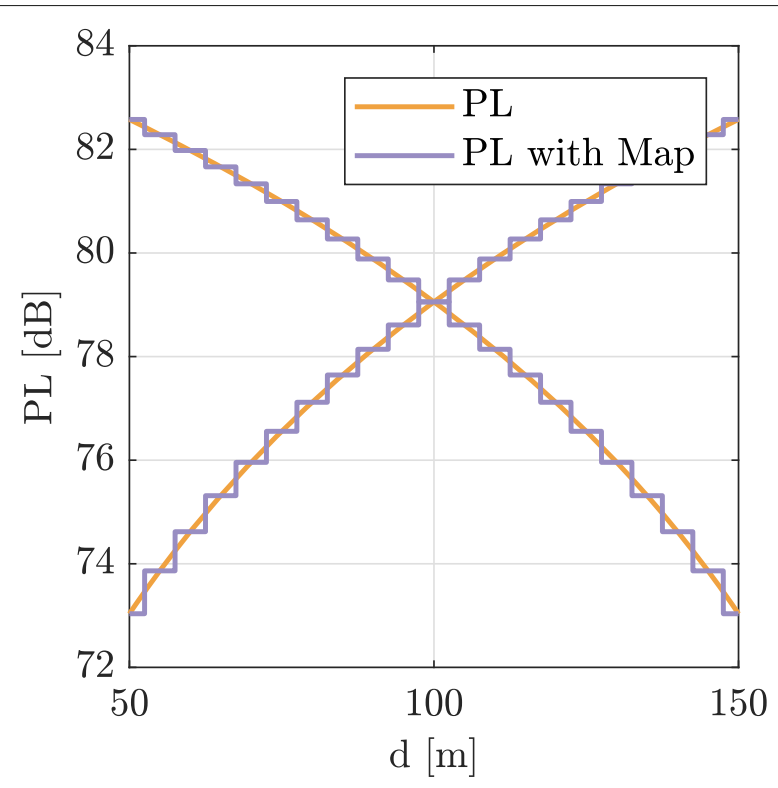

Fig. 14 Illustration of PL calculation with and without maps. In the underlying scenario, a user moves on the connecting line of two BSs. The distance between the BSs is $200 \mathrm{~m}$ and the PL model is free space with a pathloss exponent of 2 at a frequency of $2.14 \mathrm{GHz}$. The plot shows the respective $\mathrm{PL}$ values over the distance to the first BS. When using a $P L$ map with a resolution of $5 \mathrm{~m} /$ pixel there will be a region around the distance of $100 \mathrm{~m}$ where the user experiences the same PL from both BS, which leads to problems for cell association. With our precise geometry, this region is confined within a single point

inverse discrete Fourier transform can directly be used for interpolation. Also, the calculations in this step can be limited to the necessary points only, i.e., the calculation of an entire map is omitted. Thus, $b$ is a set of spatially correlated random variables where each variable corresponds to the precise position of one user. Due to this interpolation, it is also not necessary that $a$ is very large. To give an example for the necessary resolution for the uncorrelated map $a$, in [39], we interpolated a map with a resolution of $2.5 \mathrm{~m} /$ pixel and $40 \times 40$ pixel to $97 \times 97$ pixel. It is clear that the resulting autocorrelation after interpolation is not precisely the desired autocorrelation but still the errors are smaller than the errors of the used exponentially decaying model compared to real measurements [58]. Besides that, the error of the measured autocorrelation after interpolation is also smaller than the errors that are introduced by the approximation of the Cholesky decomposition in [38]. As in the Vienna LTE-A SL simulator, it is again possible to calculate different SF values for different BSs and introducing an inter-BS correlation $c$ that applies to every position. This is now done by calculating one additional spectrum $\hat{A}$ and replacing the third step by $A:=\sqrt{c} \hat{A}+\sqrt{1-c} \mathcal{F}\{a\}$.

\subsubsection{Input from Vienna 5G LL simulator}

Thanks to the applied link abstraction within our simulator and the capabilities of the Vienna 5G LL simulator, we are able to simulate transmissions with all transmit formats and parameter configurations that are available in the LL simulator. The link level performance is represented by SINR to BLER mapping tables in the LPM of the Vienna 5G SL simulator. These mapping tables have to be adapted to reflect the efficiencies of the applied modulation and coding schemes (MCSs). The SINR to BLER mapping tables are also utilized during CQI feedback calculation, to select a preferred MCS that is suitable for the current channel conditions. Supported modulation methods range from classical cyclic prefix orthogonal frequency division multiplexing (OFDM) over filtered OFDM to newly defined schemes such as universal filtered multicarrier (UFMC) or filter-bank multicarrier (FBMC). Implemented coding schemes are turbo, low-density parity-check (LDPC), polar, and convolutional coding. For any arbitrary MCS, an BLER over SINR curve can be generated by the Vienna 5G LL simulator and serve as input to the LPM. This provides an extensive freedom for simulating arbitrary combinations of modulation scheme (including modulation order) and coding scheme (including coding rate). An example for this different behavior is shown in Fig. 15 for CQIs according to the LTE-A standard and different coding schemes. These results show the necessity for adapting the LPM input.

\section{Conclusions}

For the increased diversity of scenarios and use cases, that is anticipated for $5 \mathrm{G}$ networks, a vast number of parameter combinations needs to be employed in SL simulations.

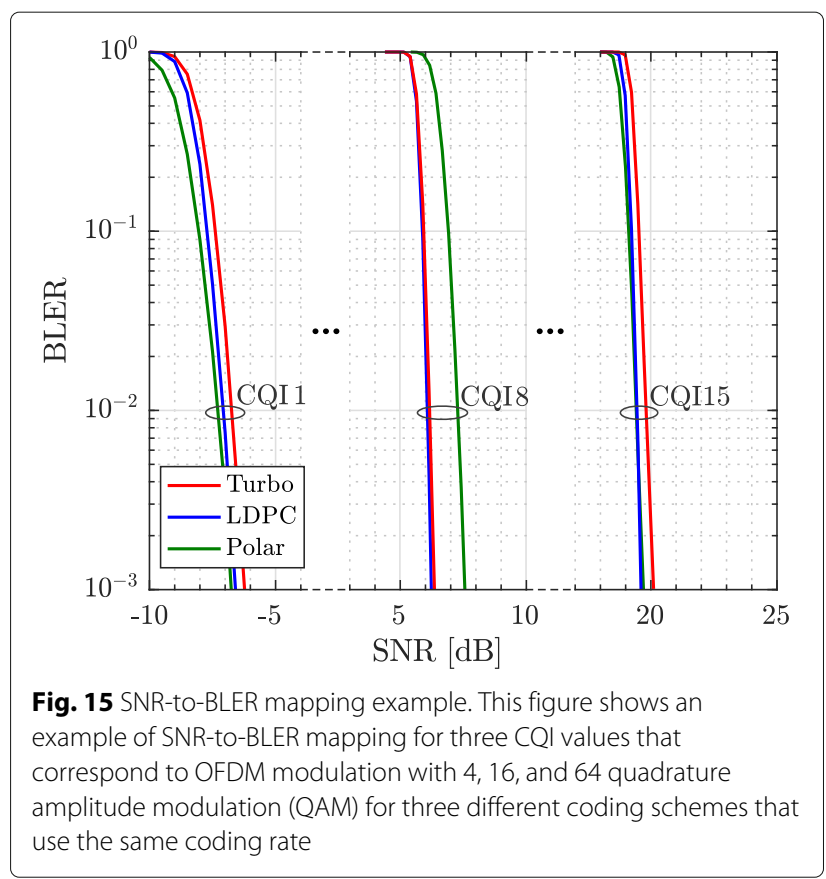


With the Vienna 5G SL simulator, we developed a tool for researchers to investigate important aspects of future networks, with the focus on multi-tier, heterogeneous networks, in combination with a substantial collection of propagation models. The relation of the path loss model choice with the actual link condition further increases the versatility of simulations and the range of potential use cases.

As can be seen from the presented structure of the simulator, it is effortlessly possible to enhance the already existing functionality and also to speed up the simulations through parallelization of the main simulation loop. Due to efficient implementation, it is possible to simulate large-scale networks with several BS and user types and several thousand active network nodes in the same simulation, without the need for specialized simulation hardware.

Since the simulator code is freely available under an academic license, we encourage active input from researchers around the world, also through our online-forum [59], and will constantly improve and extend the simulator's functionality. Thus, we establish the Vienna 5G SL simulator as a reliable and resourceful tool for mobile communications research.

\begin{abstract}
Abbreviations
3GPP: 3rd Generation Partnership Project; 2D: Two-dimensional; 3D: Threedimensional; 5G: Fifth generation of mobile communications; AWGN: Additive white Gaussian noise; BLER: Block error ratio; BS: Base station; CQI: Channel quality indicator; CSI: Channel state information; D2D: Device-to-device; DAS Distributed antenna system; FBMC: Filter-bank multicarrier; FDD: Frequency division duplex; GSC: Geometry-based stochastic channel; HHI: Heinrich Hertz Institute; iid: Independent and identically distributed; IoT: Internet of things; LL: Link level; LDPC: Low-density parity-check; LOS: Line-of-sight; LPM: Link performance model; LQM: Link quality model; LSP: Large-scale parameter; LTE: Long-term evolution; LTE-A: Long-term evolution-advanced; MAC: Medium access control; MCS: Modulation and coding scheme; MF: Macroscopic fading; MIMO: Multiple-input multiple-output; mmWave: Millimeter wave; MU-MIMO: Multi user MIMO; NLOS: Non line-of-sight; OFDM: Orthogonal frequency division multiplexing; OOP: Object-oriented programming; PDP: Power delay profile; PL: Distance dependent path loss; PHY: Physical; PMI: Precoding matrix indicator; PPP: Poisson point process; QAM: Quadrature amplitude modulation; QuaDRiGav: Quasi deterministic radio channel generator; RAN: Radio access network; RI: Rank indicator; ROI: Region of interest; RRH: Remote radio head; SF: Shadow fading; SINR: Signal to interference and noise ratio; SL: System level; SNR: Signal to noise ratio; SSP: Small-scale parameter; TDD: Time division duplex; TS: Time slot; TU: Typical Urban; UAV: Unmanned aerial vehicle; UFMC: Universal filtered multicarrier; V2X: Vehicle-to-everything; VCCS: Vienna cellular communications simulator; WLAN: Wireless local area network; XPR: Cross polarization power ratio; ZF: Zero forcing
\end{abstract}

\section{Funding}

This work has been funded by the Christian Doppler Laboratory for Dependable Wireless Connectivity for the Society in Motion. The financial support by the Austrian Federal Ministry for Digital and Economic Affairs and the National Foundation for Research, Technology and Development is gratefully acknowledged. The authors acknowledge the TU Wien University Library for financial support through its Open Access Funding Program.

\section{Availability of data and materials}

All data and results presented in this manuscript are reproducible by employing the Vienna $5 G$ system level simulator available under an academic use license free of charge at [22].

\section{Authors' contributions}

MM contributed to defining the simulator structure, performed the literature review and wrote the three introductory sections. FA wrote the sections on propagation models and heterogeneous networks and is in charge of implementing advanced channel models. TD implemented the MAC layer functionality and the shadow fading and contributed the description of precise geometry implementation. AF contributed the manuscript sections on IOT and general implementation and included the interference region, the LQM and the majority of the propagation models in the simulator. BR wrote the section on D2D and added V2X functionality to the code. AN contributed the section on channel interfaces that he also defined and implemented. LN started the implementation of the simulator and contributed large parts of the scenario definition and setup. SS supervises the simulator's development. MR initiated the simulator project and supervises the research. All authors read and approved the final manuscript.

\section{Competing interests}

The authors declare that they have no competing interests.

\section{Publisher's Note}

Springer Nature remains neutral with regard to jurisdictional claims in published maps and institutional affiliations.

Received: 9 May 2018 Accepted: 28 August 2018

Published online: 21 September 2018

\section{References}

1. J. C. Ikuno, M. Wrulich, M. Rupp, in IEEE Vehicular Technology Conference (VTC Spring). System level simulation of LTE networks (IEEE, Taipei, 2010)

2. L. Chen, W. Chen, B. Wang, X. Zhang, H. Chen, D. Yang, System-level simulation methodology and platform for mobile cellular systems. IEEE Commun. Mag. 49(7), 148-155 (2011)

3. S. Cho, S. Chae, M. Rim, C. G. Kang, in 2017 Ninth International Conference on Ubiquitous and Future Networks (ICUFN). System level simulation for $5 \mathrm{~g}$ cellular communication systems, (2017), pp. 296-299. https://doi.org/10. 1109/ICUFN.2017.7993797

4. A. Daeinabi, K. Sandrasegaran, X. Zhu, in Proceedings of the 8th ACM Workshop on Performance Monitoring and Measurement of Heterogeneous Wireless and Wired Networks. System level simulation to evaluate the interference in macrocell-picocell downlink systems (ACM, New York, 2013), pp. 125-132

5. M. Rupp, S. Schwarz, M. Taranetz, The Vienna LTE-Advanced Simulators: Up and Downlink, Link and System Level Simulation. 1st edn. Signals and Communication Technology. (Springer, Singapore, 2016). https://doi.org/ 10.1007/978-981-10-0617-3

6. Y. Peng, H. Liu, W. Liu, J. Wang, D. Wang, in 2016 19th International Symposium on Wireless Personal Multimedia Communications (WPMC). Design and implementation of FBMC system level simulation (IEEE, Shenzen, 2016), pp. 435-440

7. K. Min, M. Jung, S. Shin, S. Kim, S. Choi, in 2017 IEEE 85th Vehicular Technology Conference (VTC Spring). System level simulation of mmWave based mobile Xhaul networks (IEEE, Sydney, 2017), pp. 1-5

8. H. ElSawy, A. Sultan-Salem, M. S. Alouini, M. Z. Win, Modeling and analysis of cellular networks using stochastic geometry: A tutorial. IEEE Commun. Surv. Tutor. 19(1), 167-203 (2017)

9. I. Trigui, S. Affes, B. Liang, Unified stochastic geometry modeling and analysis of cellular networks in LOS/NLOS and shadowed fading. IEEE Trans. Commun. 65(12), 5470-5486 (2017)

10. L. Lu, G. Y. Li, A. L. Swindlehurst, A. Ashikhmin, R. Zhang, An overview of massive MIMO: Benefits and challenges. IEEE J. Sel. Top. Sig. Process. 8(5), 742-758 (2014)

11. E. Larsson, O. Edfors, F. Tufvesson, T. Marzetta, Massive MIMO for next generation wireless systems. IEEE Commun. Mag. 52(2), 186-195 (2014)

12. H. Ji, Y. Kim, J. Lee, E. Onggosanusi, Y. Nam, J. Zhang, B. Lee, B. Shim, Overview of full-dimension MIMO in LTE-advanced pro. IEEE Commun. Mag. PP(99), 2-11 (2016)

13. A. A. Zaidi, R. Baldemair, H. Tullberg, H. Bjorkegren, L. Sundstrom, J. Medbo, C. Kilinc, I. D. Silva, Waveform and numerology to support $5 \mathrm{G}$ services and requirements. IEEE Commun. Mag. 54(11), 90-98 (2016) 
14. P. Guan, D. Wu, T. Tian, J. Zhou, X. Zhang, L. Gu, A. Benjebbour, M. Iwabuchi, Y. Kishiyama, 5G field trials: OFDM-based waveforms and mixed numerologies. IEEE J. Sel. Areas Commun. 35(6), 1234-1243 (2017)

15. K. S. Ali, H. Elsawy, A. Chaaban, M. S. Alouini, Non-orthogonal multiple access for large-scale $5 \mathrm{G}$ networks: Interference aware design. IEEE Access. 5, 21204-21216 (2017)

16. Z. Ding, X. Lei, G. K. Karagiannidis, R. Schober, J. Yuan, V. K. Bhargava, A survey on non-orthogonal multiple access for $5 \mathrm{G}$ networks: Research challenges and future trends. IEEE J. Sel. Areas Commun. 35(10), 2181-2195 (2017)

17. W. Roh, J. Y. Seol, J. Park, B. Lee, J. Lee, Y. Kim, J. Cho, K. Cheun, F. Aryanfar, Millimeter-wave beamforming as an enabling technology for $5 \mathrm{G}$ cellular communications: theoretical feasibility and prototype results. IEEE Commun. Mag. 52(2), 106-113 (2014)

18. R. W. Heath, N. González-Prelcic, S. Rangan, W. Roh, A. M. Sayeed, An overview of signal processing techniques for millimeter wave MIMO systems. IEEE J. Sel. Top. Signal Proc. 10(3), 436-453 (2016)

19. S. Schwarz, J. C. Ikuno, M. Simko, M. Taranetz, Q. Wang, M. Rupp, Pushing the limits of LTE: A survey on research enhancing the standard. IEEE Access. 1, 51-62 (2013). https://doi.org/10.1109/ACCESS.2013.2260371

20. M. Taranetz, T. Blazek, T. Kropfreiter, M. K. Müller, S. Schwarz, M. Rupp, Runtime precoding: Enabling multipoint transmission in LTE-advanced system level simulations. IEEE Access. 3, 725-736 (2015)

21. E. Zöchmann, S. Schwarz, S. Pratschner, L. Nagel, M. Lerch, M. Rupp, Exploring the physical layer frontiers of cellular uplink. EURASIP J. Wirel. Commun. Netw. 2016(1), 1-18 (2016). https://doi.org/10.1186/s13638016-0609-1

22. Institute of Telecommunications, T. U. Wien, Vienna Cellular Communications Simulators. www.tc.tuwien.ac.at/vccs/. Accessed 13 Apr 2018

23. C. Mehlführer, J. C. Ikuno, M. Simko, S. Schwarz, M. Rupp, The Vienna LTE simulators - enabling reproducibility in wireless communications research. EURASIP J. Adv. Signal Proc. (JASP) Spec. Issue Reproducible Res. 2011(1), 1-14 (2011)

24. G. Piro, L. A. Grieco, G. Boggia, F. Capozzi, P. Camarda, Simulating Ite cellular systems: An open-source framework. IEEE Trans. Veh. Technol. 60(2), 498-513 (2011). https://doi.org/10.1109/TVT.2010.2091660

25. A. Virdis, G. Stea, G. Nardini, in Simulation and Modeling Methodologies, Technologies and Applications, ed. by M. S. Obaidat, T. Ören, J. Kacprzyk, and J. Filipe. Simulating Ite/lte-advanced networks with simulte (Springer, Cham, 2015), pp. 83-105

26. M. Mezzavilla, M. Zhang, M. Polese, R. Ford, S. Dutta, S. Rangan, M. Zorzi, End-to-end simulation of $5 \mathrm{~g}$ mmwave networks. CoRR. abs/1705.02882 (2017). 1705.02882

27. N. Nikaein, M. K. Marina, S. Manickam, A. Dawson, R. Knopp, C. Bonnet, OpenAirlnterface: A flexible platform for $5 \mathrm{G}$ research. ACM SIGCOMM Comput. Commun. Rev. 44(5), 33-38 (2014)

28. K. Bakowski, M. Rodziewicz, P. Sroka, in 2015 International Symposium on Wireless Communication Systems (ISWCS). System-level simulations of selected aspects of $5 \mathrm{G}$ cellular networks, (2015), pp. 711-715. https://doi. org/10.1109/ISWCS.2015.7454442

29. X. Wang, Y. Chen, Z. Mai, in 2017 IEEE Globecom Workshops (GC Wkshps). A novel design of system level simulator for heterogeneous networks, (2017), pp. 1-6. https://doi.org/10.1109/GLOCOMW.2017.8269059

30. N. Mohsen, K. S. Hassan, in 2015 IEEE 11th International Conference on Wireless and Mobile Computing, Networking and Communications (WiMob). C-ran simulator: A tool for evaluating $5 \mathrm{~g}$ cloud-based networks system-level performance, (2015), pp. 302-309. https://doi.org/10.1109/ WiMOB.2015.7347976

31. M. Liu, P. Ren, Q. Du, W. Ou, X. Xiong, G. Li, in 2016 IEEE/CIC International Conference on Communications in China (ICCC). Design of system-level simulation platform for $5 \mathrm{~g}$ networks, (2016), pp. 1-6. https://doi.org/10. 1109/ICCChina.2016.7636796

32. Y. Wang, J. Xu, L. Jiang, Challenges of system-level simulations and performance evaluation for $5 \mathrm{G}$ wireless networks. IEEE Access. 2 1553-1561 (2014). https://doi.org/10.1109/ACCESS.2014.2383833

33. 3rd Generation Partnership Project (3GPP), Technical Specification Group Radio Access Network; NR; Physical channels and modulation TS 38.211, 3GPP (2017)

34. 3rd Generation Partnership Project (3GPP), Study on 3D channel model for LTE. TR 36.873, 3GPP (2015)
35. 3rd Generation Partnership Project (3GPP), Technical Specification Group Radio Access Network; Study on channel model for frequencies from 0.5 to $100 \mathrm{GHz}$. TR 38.901, 3GPP (2017)

36. 3rd Generation Partnership Project (3GPP), Study on enhanced LTE support for aerial vehicles. TR 36.777, 3GPP (2017)

37. S. Jaeckel, et al., QuaDRiGa: A 3-D multi-cell channel model with time evolution for enabling virtual field trials. IEEE Trans. Antennas Propag. 62(6), 3242-3256 (2014). https://doi.org/10.1109/TAP.2014.2310220

38. H. Claussen, in 2005 IEEE 16th International Symposium on Personal, Indoor and Mobile Radio Communications. Efficient modelling of channel maps with correlated shadow fading in mobile radio systems, vol. 1, (2005), pp. 512-516. https://doi.org/10.1109/PIMRC.2005.1651489

39. T. Dittrich, M. Taranetz, M. Rupp, in WSA 2017; 21th International ITG Workshop on Smart Antennas. An efficient method for avoiding shadow fading maps in system level simulations (IEEE, Berlin, 2017), pp. 1-8

40. J. C. Ikuno, System level modeling and optimization of the LTE downlink. PhD thesis, E389, TU Wien (2013)

41. M. K. Müller, M. Meidlinger, M. Rupp, in IEEE 8th Sensor Array and Multichannel Signal Processing Workshop (SAM'2014). Correlated UE impairments in ZF MU-MIMO transmissions (IEEE, A Coruña, 2014), pp. 317-320

42. S. Schwarz, in Advances in Mobile Computing and Communications: $4 \mathrm{G}$ and Beyond, ed. by M. Bala Krishna, J. Lloret Mauri. Limited feedback for 4G and beyond (CRC Press Taylor \& Francis Group, Boca Raton, 2016)

43. M. Ding, D. López-Pérez, in 201715 th International Symposium on Modeling and Optimization in Mobile, Ad Hoc, and Wireless Networks (WiOpt). On the performance of practical ultra-dense networks: The major and minor factors, (2017), pp. 1-8. https://doi.org/10.23919/WIOPT.2017.7959926

44. M. Taranetz, M. K. Müller, A survey on modeling interference and blockage in urban heterogeneous cellular networks. EURASIP J. Wirel. Commun. Netw. 2016(1), 252 (2016). https://doi.org/10.1186/s13638-016-0740-z

45. ITU, Recommendation ITU-R M.1225: Guidelines for evaluation of radio transmission technologies for IMT-2000. Technical report, ITU (1997)

46. 3GPP Technical Specification Group Radio Access Networks; Deployment aspects (Release 8). (2008). [Online]. Available: http://www.3gpp.org/ftp/ Specs/html-info/25943.htm. Accessed June 2018

47. Y. R. Zheng, C. Xiao, Simulation models with correct statistical properties for rayleigh fading channels. IEEE Trans. Commun. 51(6), 920-928 (2003). https://doi.org/10.1109/TCOMM.2003.813259

48. WINNER II WP1, WINNER II channel models. IST-4-027756 WINNER I| Deliverable D1.1.2 (2007)

49. F. Ademaj, M. Taranetz, M. Rupp, 3GPP 3D MIMO channel model: A holistic implementation guideline for open source simulation tools. EURASIP J. Wirel. Commun. Netw. 2016(1), 55 (2016). https://doi.org/10. 1155/2007/19070

50. F. Ademaj, M. K. Müller, S. Schwarz, M. Rupp, in 2017 IEEE 86th Vehicular Technology Conference (VTC-Fall). Modeling of Spatially Correlated Geometry-Based Stochastic Channels, (2017), pp. 1-6. https://doi.org/10. 1109/NTCFall.2017.8287884

51. T. Berisha, C. F. Mecklenbräuker, in 2017 IEEE Vehicular Networking Conference (VNC). 2D LOS/NLOS urban maps and LTE MIMO performance evaluation for vehicular use cases, (2017), pp. 291-294. https://doi.org/10. 1109/NNC.2017.8275607

52. M. Gasser, Simulating Vehicle-to-Vehicle Connectivity on Real-World Street Networks, (2017)

53. M. K. Müller, M. Taranetz, M. Rupp, Analyzing wireless indoor communications by blockage models. IEEE Access. 5, 2172-2186 (2016)

54. S. Schwarz, M. Rupp, Society in motion: challenges for LTE and beyond mobile communications. IEEE Commun. Mag. 54(5), 76-83 (2016). https://doi.org/10.1109/MCOM.2016.7470939

55. Y. Wang, K. Venugopal, A. F. Molisch, R. W. Heath, in 2016 IEEE 84th Vehicular Technology Conference (VTC-Fall). Analysis of Urban Millimeter Wave Microcellular Networks, (Montreal, 2016), pp. 1-5. https://doi.org/ 10.1109/NTCFall.2016.7880906

56. D. Niyato, M. Maso, D. I. Kim, A. Xhafa, M. Zorzi, A. Dutta, Practical perspectives on iot in $5 \mathrm{~g}$ networks: From theory to industrial challenges and business opportunities. IEEE Commun. Mag. 55(2), 68-69 (2017). https://doi.org/10.1109/MCOM.2017.7842414

57. A. V. Kini, M. Hosseinian, M. I. Lee, J. Stern-Berkowitz, in 2015 IEEE Wireless Communications and Networking Conference (WCNC). Reevaluating cell 
wraparound techniques for 3D channel model based system-level simulations (IEEE, Boca Raton, 2015). https://doi.org/10.1109/wcnc.2015 7127464

58. M. Gudmundson, Correlation model for shadow fading in mobile radio systems. Electron. Lett. 27(23), 2145-2146 (1991). https://doi.org/10.1049/ el:19911328

59. Institute of Telecommunications, T. U. Wien, Vienna Cellular Communications Simulators Forum. www.nt.tuwien.ac.at/forum/. Accessed 13 Apr 2018

\section{Submit your manuscript to a SpringerOpen ${ }^{\odot}$ journal and benefit from:}

- Convenient online submission

- Rigorous peer review

- Open access: articles freely available online

- High visibility within the field

- Retaining the copyright to your article

Submit your next manuscript at $\gg$ springeropen.com 